Annuaire suisse de politique de développement

13 | 1994

Annuaire Suisse - Tiers Monde 1994

\title{
L'appui de la Confédération suisse au programme de redressement économique du Ghana
}

Antoine Brawand

\section{(2) OpenEdition}

Édition électronique

URL : http://journals.openedition.org/aspd/1066

DOI : 10.4000/aspd.1066

ISSN : 1663-9669

Éditeur

Institut de hautes études internationales et du développement

\section{Édition imprimée}

Date de publication : 1 janvier 1994

Pagination : 269-284

ISSN : 1660-5934

\section{Référence électronique}

Antoine Brawand, «L'appui de la Confédération suisse au programme de redressement économique du Ghana », Annuaire suisse de politique de développement [En ligne], 13 | 1994, mis en ligne le 19 décembre 2012, consulté le 08 septembre 2020. URL : http://journals.openedition.org/aspd/1066 DOI : https://doi.org/10.4000/aspd.1066 


\title{
L'appui de la Confédération suisse au programme de redressement économique du Ghana
}

\author{
Antoine Brawand
}

\section{Introduction}

Au cours des années quatre-vingt, la Confédération a participé toujours davantage aux programmes d'ajustement structurel, principalement en Afrique subsaharienne. Depuis 1992, elle met particulièrement l'accent sur l'ajustement du secteur financier. (1)

Ce type de financement est accordé en majeure partie par l'Office fédéral des affaires économiques extérieures (OFAEE). Cependant, depuis quelques années, la Direction de la coopération au développement et de l'aide humanitaire (DDA) appuie aussi certains programmes d'ajustement, généralement dans des pays qui bénéficient déjà de son aide bilatérale. Elle a aussi commencé, en coordination avec la Banque mondiale, à financer une partie de l'assistance technique destinée à la restructuration du secteur financier.

L'administration fédérale considère que les ajustements structurels représentent actuellement une stratégie appropriée pour surmonter la crise économique dans les pays en développement. Elle a constaté des résultats positifs. Toutefois, elle estime qu'il est "sans doute encore trop tôt pour se prononcer de manière définitive sur l'efficacité des programmes d'ajustement". (2)

L'étude de l'appui que la Confédération a donné au programme de redressement économique (PRE) du Ghana, entre1984 et 1992, a pour but de contribuer à la réflexion sur ce thème.

La Suisse a été l'un des premiers pays à soutenir ce programme et le Ghana a été longtemps le principal destinataire des aides suisses à la balance des paiements. L'OFAEE lui a accordé six contributions. Administrées par l'Associa- 
tion Internationale pour le Développement (IDA), les deux dernières contributions devaient également servir à soutenir la réforme du secteur financier. De son côté, la DDA a payé des consultants mis à la disposition de la Banque (centrale) du Ghana. Ce faisant, la coopération suisse a abordé un nouveau champ d'action.

Nous analyserons d'abord le PRE, ses forces et ses faiblesses, ainsi que le rôle de la réforme du secteur bancaire. Nous examinerons ensuite les objectifs, les caractéristiques et les résultats des contributions fédérales. Enfin, nous essayerons de tirer quelques leçons de l'expérience ghanéenne.

\section{Le programme de redressement économique du Ghana (3)}

Relativement prospère dans les années cinquante, le Ghana a cherché, après avoir obtenu l'indépendance politique, à se libérer de sa dépendance économique. L'échec de cette tentative a été suivi d'une longue période marquée par l'instabilité politique et le déclin de l'économie.

Fin 1981, le Provisional National Defence Council (PNDC) dirigé par le lieutenant d'aviation J. Rawlings s'empara du pouvoir. II suspendit la Constitution, dissout le Parlement et abolit les partis politiques. Sa rhétorique révolutionnaire indisposa les milieux financiers internationaux et la bourgeoisie locale. La crise financière de l'Etat s'aggrava. D'autre part, en 1982-83, les termes de l'échange s'effondrèrent, le Nigeria renvoya un million de Ghanéens et une longue sécheresse provoqua la famine dans tout le pays.

\subsection{Principales mesures économiques, $1984-92$}

Les courants de gauche et les partisans d'une entente avec les institutions financières internationales s'affrontèrent. La tendance pro-occidentale l'emporta. En avril 1983, le Gouvernement adopta le Programme de redressement économique (PRE) qui avait été négocié avec le FMI et la Banque mondiale.

Pour les années 1984 à 1986 le PRE prévoyait différentes mesures:

- il s'agissait d'enrayer le long déclin de l'économie et de la libéraliser;

- la monnaie nationale (le cedi) dont le cours avait été maintenu à un niveau trop élevé devait être fortement dévaluée;

- les prix payés aux producteurs, de cacao notamment, devaient être améliorés, les subventions réduites et les infrastructures réhabilitées;

- pour mobiliser des ressources financières, le Gouvernement chercherait à retrouver la confiance des détenteurs de capitaux, tant nationaux qu'étrangers.

Dans les années 1987 à 1989, le PRE entra dans une nouvelle phase consistant à promouvoir la poursuite de la croissance (5\% par an), l'épargne et l'investissement sans négliger pour autant la lutte contre l'inflation. Le Gouvernement continua à libéraliser les transactions courantes avec l'étranger et il s'efforça d'unifier les taux de change. II entama la restructuration du secteur financier. II chercha aussi à améliorer la gestion du secteur public et à privatiser certaines entreprises. 
Les programmes adoptés depuis 1990 reprennent ces objectifs. Le Gouvernement prévoit en outre d'accélérer les privatisations et de lutter contre la pauvreté en veillant à la distribution régionale et sectorielle des dépenses gouvernementales.

Le Ghana a eu recours aux différentes formes de financement accordées par le FMI: accords de confirmation jusqu'en 1987, facilité élargie jusqu'en 1988, facilité d'ajustement renforcée de 1989 à 1991 et facilité de financement compensatoire en 1993.

Les contributions de la Banque mondiale, quant à elles, prirent de plus en plus la forme de crédits de programme. Dans un premier temps, ceux-ci servirent surtout à promouvoir la libéralisation du commerce extérieur, puis les ajustements globaux et sectoriels. Cependant, en 1992, la Banque mondiale a prévu de redonner la priorité aux financements de projets (en leur consacrant au moins $75 \%$ des nouveaux crédits). Elle considérait que les progrès accomplis permettaient désormais de réduire la part des crédits à déboursement rapide.

\subsection{Un redressement spectaculaire}

Le PIB par habitant a augmenté de $2 \%$ par an en moyenne entre 1983 et 1992 . Ce résultat contraste avec le recul observé pendant ce temps dans la plupart des pays d'Afrique subsaharienne.

Des déséquilibres macroéconomiques ont été corrigés: le déficit budgétaire ainsi que celui de la balance des paiements, l'expansion excessive de la masse monétaire et l'inflation galopante. La dette extérieure qui était relativement petite n'a pas dû être rééchelonnée et les arriérés ont été acquittés.

Ces succès ont fait apparaître l'ajustement de l'économie ghanéenne comme un cas exemplaire et les bailleurs de fonds n'ont pas manqué d'en faire état pour justifier leurs politiques.

\subsection{Analyse des causes}

En 1983, l'économie formelle du Ghana était paralysée après vingt années d'instabilité politique, d'étatisme excessif et de mauvaise gestion. Deux années consécutives de sécheresse venaient de détruire les récoltes et de provoquer la famine.

Néanmoins, le pays avait retrouvé une certaine stabilité politique. Le Gouvernement appliqua les mesures demandées par les bailleurs de fonds et obtint leur appui. De bonnes récoltes entraînèrent une certaine stabilisation des prix agricoles. La réintégration étonnamment rapide du million de Ghanéens chassés du Nigeria (dont beaucoup étaient des travailleurs expérimentés) favorisa aussi l'augmentation de la production. Grâce à l'infrastructure mise en place sous le Président K. Nkrumah, dans le domaine énergétique notamment, le besoin de nouveaux investissements ne se fit pas trop sentir. Le taux d'utilisation des capacités de production, qui était tombé très bas, remonta. L'amélioration temporaire des termes de l'échange (jusqu'en 1987) permit au Gouvernement d'augmen- 
ter la rémunération réelle des producteurs ruraux, de cacao en particulier, et de relancer les exportations.

Le Ghana a donc réussi à faire cohabiter ajustement et croissance. Grâce à des financements internationaux publics élevés, les importations se sont accrues bien plus vite que les exportations. Ainsi le Ghana a pu augmenter ses dépenses de santé et d'éducation, qui s'étaient effondrées, alors que les pays sous ajustement doivent souvent réduire les budgets sociaux. II a poursuivi cette politique de croissance alors même que les termes de l'échange, en 1988-89, se dégradaient à nouveau. Les bailleurs de fonds comblèrent le déficit de la balance commerciale non seulement pour soutenir un pays qui le méritait, mais pour sauver la renommée du modèle d'ajustement.

\subsection{Faiblesses du processus de redressement}

L'endettement extérieur a progressé de 1,4 milliards de dollars fin 1980 à 4,3 milliards fin 1992 (avances du FMI comprises). Quant au ratio du service de la dette, il est passé de $13 \%$ des exportations de biens et de services fin 1980 à $30 \%$ fin 1992. Des analystes ghanéens estiment que, compte tenu de l'importance des financements extérieurs et des facteurs qui ont favorisé le PRE, le taux de croissance du PIB a été insuffisant.

La dévaluation de la monnaie nationale (le cedi) a été et reste spectaculaire. A la fois résultat et cause de l'inflation, elle a ruiné des entreprises qui avaient contracté des emprunts en monnaie étrangère. Ces emprunts étaient généralement garantis par les banques locales.

Le Gouvernement a dû donner la priorité aux activités d'exportation. Les ventes d'or connaissent actuellement la plus grande expansion. Par contre, faute d'appuis suffisants, l'agriculture vivrière stagne. Les régions particulièrement exposées à la sécheresse (le nord surtout) connaissent des périodes de disette. L'industrie manufacturière qui travaille essentiellement pour le marché intérieur est peu soutenue. Elle accède difficilement au crédit, contrairement aux commerçants. La levée du contrôle des importations et des restrictions de change l'a fortement exposée à la concurrence étrangère. Le taux d'utilisation de la capacité de production est insuffisant.

Les investissements privés dans les activités productives restent généralement faibles, sauf dans les mines.

Pour les raisons exposées plus haut, les effets sociaux de l'ajustement ne semblent pas avoir été aussi graves qu'ailleurs. Cependant, au Ghana aussi, des subsides ont été supprimés et certains services publics sont désormais payants. Les salaires sont restés extrêmement bas. L'administration, les entreprises publiques et certaines sociétés privées ont fortement réduit leur personnel.

\subsection{Le rôle du secteur bancaire}

Les principaux problèmes du secteur financier, dans l'optique du développement et de la croissance à long terme, sont la mobilisation insuffisante de l'épargne et 
le manque de crédits aux entreprises (industrielles et agricoles) qui produisent pour le marché domestique. Avant la réforme bancaire, ces carences étaient dues à des taux d'intérêt réels souvent négatifs, à la politique monétaire restrictive et aux difficultés croissantes des banques.

Outre la banque centrale (Banque du Ghana), le secteur bancaire ghanéen comprend notamment six banques commerciales, trois banques de développement, quelques banques d'affaires (la plupart récentes) et plus de 100 banques rurales. Trois banques commerciales (dont la plus grande) sont entièrement contrôlées par l'Etat, de même que les banques de développement. II y a deux filiales de banques anglaises. Le total des actifs figurant aux bilans s'élevait à 1 milliard de dollars avant la réforme bancaire. Les banques commerciales en détenaient plus de $80 \%$.

L'Etat avait fondé les banques de développement pour favoriser l'octroi de crédits à moyen et à long terme aux secteurs productifs. II faut aussi savoir qu'une grande partie des transactions financières s'effectuent à l'extérieur du système bancaire. L'épargne est souvent thésaurisée ou placée dans des biens réels.

En 1987, la plupart des banques contrôlées par l'Etat étaient virtuellement en faillite. La plupart des débiteurs, dont beaucoup de sociétés publiques, ne pouvaient plus remplir leurs obligations. Les crédits non productifs avaient souvent été accordés sans une étude approfondie des demandes et sous pression. Cependant, la dévaluation rapide de la monnaie nationale et les difficultés de l'industrie étaient aussi responsables de la crise.

Ces constatations ont amené le Gouvernement à lancer deux programmes d'ajustement du secteur financier (FINSAP I en 1988 et FINSAP II en 1991). Le financement extérieur provenait de la Banque mondiale, ainsi que de la Banque africaine de développement, du Japon et de la Suisse. Le montant total des crédits s'est élevé à environ $\mathbf{4 4 0}$ millions de dollars.

La réforme comportait trois éléments:

- Restructuration financière: les banques ont pu échanger leurs créances non productives contre des obligations émises par la Banque du Ghana.

- Restructuration institutionnelle: la loi bancaire de 1989 fait peu de distinctions entre les banques commerciales et les banques de développement. Soumises à une supervision renforcée, les banques doivent détenir au moins $6 \%$ de fonds propres.

- Restructuration de la gestion: Début 1990, le Gouvernement a nommé de nouvelles directions dans les banques qu'il contrôlait. Elles ont reçu une assistance technique ou bénéficié de jumelages avec des banques étrangères. Elles ont réduit l'effectif du personnel d'un tiers et fermé des succursales. En 1991, l'Etat a prévu de réduire ses participations à $40 \%$ du capital des banques qu'il contrôlait entièrement. II semble toutefois qu'à fin 1993, aucune vente d'actions n'ait encore eu lieu.

II est encore trop tôt pour faire un bilan de la réforme. On peut constater des effets positifs: les banques ont amélioré la structure de leurs actifs, elles ont de 
nouveau pu annoncer des profits (après constitution des provisions désormais exigées par la Banque centrale) et elles paraissent plus efficaces.

Cependant, commel'Etat émet des bons du Trésor fortement rémunérés et que les banques ne veulent pas répéter leurs erreurs passées, elles sont maintenant très restrictives en matière de crédits lorsque les emprunteurs ne peuvent pas fournir d'excellentes garanties. Cette politique ne correspond pas aux besoins de l'agriculture et de l'industrie.

D'autre part, la Banque du Ghana a transmis les créances non productives qu'elle a rachetées aux banques à une société publique de recouvrement. Celleci donne des conseils aux débiteurs, elle leur accorde assez souvent des délais de paiement supplémentaires, mais elle ne les aide pas à se restructurer.

Enfin, à cause de la persistance de fortes disparités économiques et sociales, on peut s'interroger sur l'opportunité de conférer à tous les établissements le statut de banques universelles, et de chercher à les privatiser toutes. Lorsqu'il avait fondé les banques de développement, le Gouvernement ghanéen cherchait à compléter le système bancaire. Il est fort probable que dans quelques années, le Gouvernement sera amené à reconstituer les maillons perdus.

\subsection{Perspectives}

Exprimé en valeurs de 1989, le PIB du Ghana s'élevait à environ 550 dollars par habitant en 1965 et à 400 dollars en 1992. Si l'on retient le taux de $2 \%$ qui correspond à la croissance annuelle moyenne réalisée depuis 1983 , le Ghana retrouvera son niveau de 1965 dans une quinzaine d'années seulement. Comme les coûts d'adaptation au modèle de production et de consommation importé augmentent bien plus vite, on doit s'attendre au renforcement des inégalités et à des conflits sociaux.

Toutefois, le Gouvernement et la Banque mondiale ont conçu une série de nouvelles réformes qui permettraient d'accélérer le rythme de croissance. En appliquant la recette, le Ghana pourrait emboîter le pas aux pays d'Asie de l'Est et du Sud-Est. A ce propos, la presse gouvernementale affirmait en avril 1993 que le Ghana se trouvait désormais sur le seuil de l'industrialisation massive. Cet optimisme paraît plutôt excessif.

A cause de nouvelles sécheresses et des faiblesses exposées plus haut, la croissance annuelle du PIB par habitant est tombée à 1,5\% entre 1990 et 1992. Les estimations préliminaires pour 1993 donnent un même ordre de grandeur.

Notons aussi que faute d'une production intérieure assez diversifiée, la demande d'importations reste inélastique. Quant aux recettes d'exportation, elles restent largement tributaires des ventes de matières premières dont les cours sont instables. La balance des paiements ne peut être équilibrée qu'avec des dons et des prêts concessionnels. En juin 1993, les membres du Groupe consultatif pour le Ghana réunis à Paris sous l'égide de la Banque mondiale ont promis des contributions s'élevant à 2,1 milliards de dollars pour une période de 2 ans.

Rappelons enfin que, de 1983 à 1992, les décisions relatives au PRE ont été prises en l'absence de toute procédure de contrôle parlementaire. L'information 
était limitée et la presse gouvernementale rendait rarement compte des critiques. Cette situation facilita l'acceptation des conditions posées par les institutions financières internationales. Le Ghana s'est récemment redonné des structures démocratiques. Les bailleurs de fonds devront en tenir compte.

\section{L'appui de la Confédération suisse}

\subsection{Montants, objectifs et conditions générales de l'aide}

De 1984 à 1992, la Confédération a versé 87 millions de francs pour le redressement de l'économie ghanéenne, essentiellement sous la forme d'aides (non remboursables) à la balance des paiements. On peut distinguer trois types de contributions:

- Entre 1984 et 1988, I'OFAEE a accordé quatre aides bilatérales directes s'élevant à 53 millions de francs pour la remise en état d'équipements et le financement d'importations;

- Entre 1988 et 1991, l'OFAEE a contribué à raison de 30 millions de francs au financement de deux programmes pour l'ajustement du secteur financier entrepris par le Ghana avec le concours de l'IDA;

- La Direction de la coopération au développement et de l'aide humanitaire (DDA) a soutenu ces programmes d'ajustement par le financement de consultants mis à la disposition de la Banque (centrale) du Ghana (coût:3,6 millions de francs).

En outre, la DDA a financé la restructuration de la Croix-Rouge ghanéenne (projet appuyé par la Croix-Rouge suisse notamment), de petits projets d'approvisionnement en eau et des actions humanitaires. Pour sa part, l'OFAEE a aussi contribué à un projet de gestion des importations ghanéennes coordonné par le Centre du Commerce International CNUCED/GATT.

II convient enfin de noter une amélioration intervenue au niveau des relations économiques. En mai 1991, la Commission fédérale pour la Garantie contre les risques à l'exportation (GRE) a décidé d'entrer de nouveau en matière sur les demandes de garanties à moyen et à long termes, lorsque les débiteurs sont couverts par leur banque et par le Ministère des finances du Ghana. (4) D'autre part, en octobre 1991, un accord sur la protection des investissements a été conclu.

Les objectifs et les conditions générales des financements consentis par I'OFAEE étaient conformes à la politique d'aide à la balance des paiements exposée dans les Messages du Conseil fédéral concernant la coopération économique avec les pays en développement: (5)

- Permettre à des pays pauvres qui traversent une grave crise de "financer les importations de biens et de services indispensables à la couverture de leurs besoins essentiels ou à une meilleure utilisation des capacités de production existantes"; 
- "Les pays bénéficiaires entreprennent de sérieux efforts pour améliorer leurs conditions économiques générales et/ou leurs structures dans des secteurs spécifiques";

- L'aide limitée de la Suisse peut consentir "ne peut avoir un effet positif que si elle fait partie intégrante d'un soutien plus large de la part de la communauté internationale, c'est à dire de donateurs multilatéraux et bilatéraux".

Rappelons aussi qu'au cours des années quatre-vingt, les autorités suisses ont davantage pris conscience de la durée des ajustements structurels. Elles ont de plus en plus insisté sur la nécessité des réformes dans les pays concernés et sur le rôle des institutions de Bretton Woods.

\subsection{Contributions bilatérales directes}

La Confédération fut l'un des premiers participants bilatéraux au programme de redressement économique du Ghana. Au cours des années 80 , ce pays devint le plus grand destinataire des aides suisses à la balance des paiements. II a reçu $24 \%$ des aides accordées entre 1980 et 1989. (6)

La justification de ce choix se résume ainsi: (7) pays en développement à faible revenu, le Ghana se trouvait dans une situation critique à la suite d'un déclin économique alarmant et d'une grave sécheresse; le Gouvernement ghanéen a pris un ensemble de mesures énergiques qui ont favorisé un redressement spectaculaire; les efforts du Ghana sont soutenus par la communauté internationale, sous l'égide du FMl et de la Banque mondiale.

La coïncidence des motifs de la politique de coopération au développement avec les intérêts de la politique économique extérieure (promotion des exportations) explique l'intervention rapide et massive de la Confédération. (8) Les entreprises textiles et pharmaceutiques ghanéennes, ainsi que la Compagnie publique des transports, étaient d'excellentes clientes des fournisseurs suisses. La dégradation de la situation économique du Ghana entraîna la suspension de la Garantie fédérale contre les risques à l'exportation pour les opérations à moyen et à long termes et l'arrêt des crédits. Sans l'octroi des aides à la balance des paiements, les fournisseurs suisses auraient certainement perdu une grande partie de leurs débouchés au Ghana. Les concurrents capables de mobiliser des crédits auraient obtenu les contrats. Ne parvenant pas à se procurer les pièces de rechange indispensables, des entreprises ghanéennes auraient fini par remplacer des équipements suisses encore tout à fait valables par des installations neuves vendues à crédit.

Notons toutefois que les importateurs doivent, quant à eux, accepter des conditions de paiement souvent très rigides dans le cadre de ces aides. Sauf exception, ils doivent payer d'avance, avant la réception des marchandises ou même au moment de confirmer la commande. Les procédures tendent donc à favoriser les opérations dont le cycle est court (commerce) au détriment des opérations à cycle long (production). On comprend donc que les industriels préfèrent souvent les crédits commerciaux aux aides à la balance des paiements. 
Voici les principales caractéristiques des aides à la balance des paiements accordées au Ghana dans le cadre de la coopération bilatérale directe: (9)

Aide I:

Accord du 11-4-1984

Montant: 12,7 millions de francs

Biens financés: Pièces de rechange et équipements accessoires destinés à la réhabilitation du parc de camions Saurer de la State Transport Corporation (STC); assistance technique.

Objectifs: A cause du manque de pièces détachées et d'une mauvaise gestion, la plupart des véhicules de la compagnie étaient tombés en panne. L'aide avait pour but de les remettre en circulation afin d'assurer le transport des carburants et la distribution des marchandises (notamment l'évacuation des récoltes de cacao). L'entreprise avait l'obligation de payer les pièces de rechange en monnaie locale. Pour sa part, le Gouvernement s'engageait à employer ces recettes dans le cadre du Programme public d'investissements.

Résultats: L'aide de la Suisse a permis de rétablir rapidement une bonne partie de la capacité de transport de la STC et d'améliorer ainsi l'approvisionnement local et l'évacuation des récoltes. Cependant, après l'achèvement de l'assistance technique (2 ans), la majorité des camions sont peu à peu retombés en panne à cause d'une nouvelle pénurie en pièces de rechange. (10)

Remarque: Deux facteurs ont joué un rôle déterminant dans l'octroi de cette première aide à la balance des paiements. D'abord, l'incapacité de la STC de répondre à la demande de transports avait des conséquences dramatiques pour les producteurs et les consommateurs ghanéens; en outre, le pays perdait des recettes d'exportation. Ensuite, étant équipée en camions Saurer, la STC avait besoin de pièces de rechange et d'assistance technique de cette firme suisse.

En Suisse, la poursuite de la production des camions Saurer était menacée. Le Conseil fédéral a donc à la fois aidé le Ghana et rendu service à l'entreprise Saurer par l'octroi de cette aide liée. Le montant de 12,7 millions de francs fut prélevé sur un crédit complémentaire ouvert en 1983 dans le cadre d'une série de mesures visant le renforcement de l'économie suisse.

Aide II:

Accord du 23-7-1985

Montant: 20 millions de francs

\section{Biens financés:}

Matières premières, pièces de rechange et équipements de remplacement destinés aux secteurs suivants:

- industrie textile millions de francs

- transports routiers

12,1

- transports ferroviaires

- industrie pharmaceutique 
Objectifs: Continuer à soutenir le redressement économique du Ghana, compte tenu des priorités indiquées par le Gouvernement, des apports d'autres bailleurs de fonds et des possibilités de passation de marchés. Les entreprises textiles ghanéennes sont en partie équipées de machines suisses et elles se sont toujours approvisionnées en pièces de rechange, en colorants et en produits chimiques dans notre pays. L'industrie pharmaceutique est aussi un client traditionnel. Quant aux transports routiers, il s'agissait de poursuivre l'approvisionnement de la STC en pièces de rechange. Comme lors de la première aide, les bénéficiaires des allocations de devises devaient en verser la contrevaleur sur un compte spécial du Gouvernement destiné au financement du Programme public d'investissements.

Résultats: La plupart des entreprises ont rapidement utilisé leurs allocations. L'aide a favorisé le redémarrage de la production de textiles et de médicaments. Certaines entreprises n'ont toutefois pas réussi à faire face au brusque renchérissement des importations provoqué par la dévaluation spectaculaire de la monnaie nationale (50\% en 6 mois). Les entreprises de transports routiers et ferroviaires en particulier éprouvèrent beaucoup de difficultés à réunir les fonds de contrevaleur et ne purent pas disposer des pièces de rechange à temps.

Remarque: Ayant été attribuée essentiellement à des secteurs industriels et à des entreprises qui étaient des clients traditionnels de fournisseurs suisses, cette seconde aide était à son tour partiellement liée. Cependant, les bénéficiaires ghanéens avaient l'obligation, lorsque c'était possible, de transmettre à l'OFAEE trois devis provenant de fournisseurs différents (dont une firme suisse) avant de passer leurs commandes.

Vu les distorsions de la concurrence entraînées par la pratique des aides liées, cette procédure se justifiait sans doute. Dans les branches prévues dans l'accord d'aide, les fournisseurs suisses paraissaient compétitifs. D'autre part, le redémarrage d'entreprises textiles et pharmaceutiques approvisionnant le marché local en biens essentiels faisait partie du Programme de redressement économique.

Aide III:

Accord du 9-12-1986 Montant: 10 millions de francs

Biens financés: Matières premières, pièces de rechange et équipements de remplacement destinés aux secteurs suivants:

- industrie textile

millions de francs

- industrie pharmaceutique

3,8

- matériel scolaire

3,8

- ascenseurs d'hôpitaux

Objectifs: Améliorer notamment l'approvisionnement des industries textiles et pharmaceutiques, clientes traditionnelles de fournisseurs suisses, afin d'accroître 
l'utilisation des capacités de production et, par conséquent d'augmenter l'offre locale de tissus et de médicaments.

Résultats: La majeure partie du crédit a pu être utilisée conformément aux objectifs. Certaines entreprises ont toutefois dû attendre assez longtemps avant d'obtenir les garanties bancaires nécessaires à la passation des commandes. D'autres n'ont pas réussi à utiliser leur allocation en devise à cause de leurs difficultés financières et commerciales. Celles-ci étaient dues, en partie, à l'absence d'une politique dynamique dans les domaines industriel et bancaire.

Remarque: voir Aide II.

Aide IV:

Accord du 12-12-1988

Montant: 10 millions de francs

Biens financés: Importations de marchandises par les entreprises qui ont acquis des devises vendues aux enchères par la Banque du Ghana.

Les contrevaleurs en monnaie locale ont été versées sur un compte destiné à financer quelques éléments d'un vaste projet entrepris par le Ghana avec le soutien de ses principaux donateurs: le Programme d'actions visant à atténuer les coûts sociaux de l'ajustement (PAMSCAD).

Objectifs: Les ventes aux enchères de devises devaient favoriser la libéralisation du régime des importations et de celui des changes. Les devises procurées par la Suisse étaient toutefois destinées au financement de livraisons de biens prioritaires facturées en francs suisses.

Les actions soutenues par la Suisse dans le cadre du PAMSCAD concernaient essentiellement les régions défavorisées du nord: approvisionnement en eau potable grâce au creusement de puits, financement de petites entreprises artisanales et programme d'alphabétisation notamment.

Résultats: Le système des ventes aux enchères de devises a favorisé l'utilisation rapide du crédit et en a facilité la gestion. Un petit nombre d'entreprises textiles et alimentaires ont obtenu la majeure partie des devises procurées par la Confédération. II s'agissait d'entreprises relativement prospères, disposant de bonnes relations avec les banques commerciales et clientes traditionnelles des fournisseurs suisses.

Les résultats du PAMSCAD ont été décevants. L'administration ghanéenne n'avait pas la capacité de suivre un grand nombre d'actions tout en respectant les conditions multiples posées par les donateurs. Le fonds de contrevaleur de l'aide IV n'a été utilisé qu'en partie. (11)

Remarque: Le remplacement du système d'allocations des devises fondé sur l'estimation des besoins par le mécanisme des ventes aux enchères a notamment entraîné les effets suivants: Une moindre visibilité de la contribution suisse et la 
suppression des appuis donnés précédemment (par la liaison partielle des aides) aux exportateurs suisses de camions, de machines textiles, de produits chimiques et de composants pharmaceutiques. (12) Les cas d'entreprises ghanéennes qui ne parvenaient pas à utiliser leur allocation en devise ont disparu. Cela a permis d'accélérer le déboursement de l'aide, mais il serait utile de savoir ce que ces entreprises sont devenues.

\subsection{Cofinancements et assistance technique pour la restructuration bancaire}

\section{Montants des cofinancements}

En août 1988 et en octobre 1991, l'OFAEE a accordé au Ghana deux nouvelles aides à la balance des paiements de 15 millions de francs chacune. Ces contributions bilatérales non remboursables ont complété les crédits de l'IDA pour l'ajustement du secteur financier.

\section{Biens financés}

Administrée par l'IDA, la contribution suisse devait servir au financement d'importations de biens et de services indispensables au bon fonctionnement de l'économie ghanéenne.

L'IDA a effectué des tirages sur un compte ouvert par la Confédération au terme de la procédure suivante:

- L'importateur ghanéen achète des devises lors des ventes aux enchères hebdomadaires effectuées par la Banque du Ghana. (Ce système a été remplacé au printemps 1990 par le marché interbancaire.) La loi de l'offre et de la demande intervient donc dans cette première phase de sélection des importations.

- La Banque du Ghana demande l'autorisation de tirer sur une ligne de crédit administrée par l'IDA. Celle-ci accepte lorsque les importations prévues correspondent à la liste des biens et services agréée par elle et par le bailleur de fonds. La liste de I'IDA est très large. Quant à la Confédération, elle a exclu le financement de trois catégories de biens: les engrais, les aliments et les carburants.

\section{Utilisation des contrevaleurs}

Les versements des importateurs ont alimenté un compte tenu par la Banque du Ghana. Dans le cadre du premier programme d'ajustement du secteur financier, ce compte a servi à la reconstitution d'actifs appartenant aux banques en difficultés. Dans le cadre du deuxième programme, il a financé le budget de l'Etat. Rappelons que le FMI et la Banque mondiale surveillent ce budget dans le cadre des accords concernant l'ajustement structurel. 


\section{Objectifs}

Dans leurs exposés des motifs, les autorités suisses fournissent des analyses et préconisent des remèdes qui sont en accord avec les principes de la Banque mondiale. Concernant le premier cofinancement, la Confédération a insisté sur les points suivants: les banques ghanéennes ont beaucoup souffert de la dégradation des activités productives; elles n'ont pas cherché à mobiliser des ressources propres et à prêter à long terme; les réformes visent à développer un secteur financier efficace, doté de banques capables de répondre aux besoins de l'économie; ce programme est indispensable au redressement économique à long terme du Ghana. (13)

Concernant le deuxième cofinancement, l'OFAEE a noté que le Ghana avait réussi à réduire considérablement le poids du service de la dette. Pour que ce résultat positif ne le pénalise pas au niveau de l'attribution de l'aide, la Confédération a fait bénéficier le Ghana des mesures complémentaires de désendettement. (14) En ce qui concerne les objectifs, la Confédération a insisté sur le renforcement de la Banque centrale et sur la continuation de la restructuration des autres banques. A long terme, les autorités suisses espèrent que ces efforts favoriseront un "développement auto-centré durable". (15)

Le déblocage des contributions suisses fut soumis, de facto, aux conditions de I'IDA. Les tranches de la première contribution suisse furent libérées en même temps que celles du crédit de l'IDA. La deuxième contribution suisse fut toutefois libérée entièrement au moment du premier versement de l'IDA. Dans ce cas, la conditionnalité suisse a donc été un peu moins sévère.

\section{Assistance technique}

La Confédération a complété ses deux cofinancements par une assistance technique. De 1989 à 1992, la DDA a financé l'intervention d'un groupe de consultants chargé d'assister la Banque du Ghana au niveau de la restructuration bancaire. Ceux-ci ont notamment formulé le cadre général de la réforme, préparé les plans de restructuration de quatre banques commerciales et de trois banques de développement et suivi la mise en oeuvre de ces plans.

Le groupe de consultants était subordonné au Gouverneur de la Banque du Ghana. Ils ont régulièrement informé la DDA, en termes généraux, de l'avancement de leur mission.

\section{Résultats}

Les résultats de l'aide suisse correspondent à ceux des deux programmes d'ajustement du secteur financier (voir point 2.5). L'appui suisse est moins identifiable qu'auparavant. La population ghanéenne n'en a généralement pas connaissance. D'autre part, des exportateurs suisses regrettent le temps des trois premières aides bilatérales directes qui leur ont souvent facilité la tâche. Leurs regrets sont d'autant plus vifs que d'autres pays continuent à fournir des financements liés! 


\section{Conclusion}

L'expérience ghanéenne est riche en leçons concernant l'appui aux programmes d'ajustement structurel et les modalités d'intervention de la coopération suisse.

Des circonstances favorables et une grande persévérance ont permis au Ghana de concilier ajustement et croissance. Toutefois, l'insistance sur les performances macroéconomiques occulte souvent les problèmes concrets que le pays rencontre aux niveaux de la production de biens et services essentiels et de la répartition des revenus. Les perspectives d'amélioration des niveaux de vie ne sont pas brillantes et beaucoup de Ghanéens parlent avec nostalgie des années cinquante et soixante relativement prospères.

La persistance de ces problèmes montre que le respect des conditions fixées dans les programmes d'ajustement structurel ne représente pas une garantie suffisante de l'utilisation efficace des contributions bilatérales. Rappelons que la Confédération a soutenu le programme de redressement économique du Ghana dans les domaines suivants: les trois premières aides suisses ont été consacrées à des importations nécessaires à la production locale de biens et de services; la quatrième aide a alimenté le système de vente aux enchères de devises et les contrevaleurs en monnaie locale ont servi à financer un programme d'atténuation de la pauvreté; gérées par l'IDA, les deux dernières aides ont servi à cofinancer le programme d'ajustement du secteur financier. Elles ont été complétées par une assistance technique.

Le financement d'importations nécessaires à la production locale de biens et de services a eu un impact positif. On pourrait certes critiquer le choix de secteurs et d'entreprises où les fournisseurs suisses étaient généralement bien placés, mais les évaluations concernant l'aide au Ghana n'ont pas révélé d'importantes distorsions de la concurrence. Par contre, certaines opérations de réhabilitation auraient mérité d'être mieux préparées et suivies.

Quant au système de vente aux enchères de devises, il n'avait pas pour but d'assurer la sélection d'importations essentielles. II a favorisé la mise en place ultérieure d'un marché interbancaire. L'affectation des contrevaleurs en monnaie locale à un programme d'atténuation de la pauvreté n'a pas été une solution satisfaisante parce que celui-ci était mal intégré au programme de redressement économique.

En fournissant un cofinancement pour le programme d'ajustement du secteur financier, la Confédération a été associée dans une certaine mesure aux discussions de la stratégie. On ne peut pas encore dresser un bilan de la réforme. Cependant, il est à craindre que certains objectifs restent hors de portée. La réforme devait permettre aux banques de répondre aux besoins de l'économie et de contribuer au redressement. Les banques ont été assainies, mais elles ne remplissent pas encore correctement ce rôle. Pour qu'elles puissent le faire, le Gouvernement devrait d'ailleurs assouplir sa politique monétaire.

Pour accroître son efficacité et la qualité de ses interventions, la coopération suisse doit continuer à viser des domaines clés du redressement économique des pays en développement, renforcer sa présence sur le terrain et appuyer très 
concrètement les efforts des acteurs locaux. En ce qui concerne le secteur financier ghanéen, la Confédération devrait favoriser l'octroi de crédits aux entreprises qui produisent des biens essentiels pour le pays et pour l'exportation, source de devises indispensables. Dans ce but, elle devrait financer la création de fonds de développement et accorder une nouvelle assistance technique.

\section{Notes :}

1. DDA et OFAEE, Mémorandum 1992 de la Suisse au Comité d'aide au développement de I'OCDE, 16-7-1993, pp. 26-27.

2. Coopération au développement de la Confédération Suisse, Rapport 1991, Berne, 1992, p.2

3. On trouvera une présentation plus étendue dans "Le redressement économique du Ghana: un processus exemplaire?", Informations et Commentaires, Revue Internationale de Sciences Sociales Appliquées (Villeurbanne, France), No 88 (spécial Afrique), juillet-septembre 1994, à paraître.

4. ERG Aktuell, No 13, juin 1991, p.1.

5. Messages concernant la continuation du financement de mesures de politique économique et commerciale au titre de la coopération internationale au développement, 1981, 1986 et 1990. Les citations qui suivent sont tirées du Message du 21-2-1990, pp.74-76.

6. Idem, p.129.

7. Message du 19-2-1986, pp.143-146; Message du 21-2-1990, pp.132-136.

8. Voir l'excellente analyse publiée à ce propos par la Commission nationale suisse Justice et Paix: Coopération au développement et intérêts économiques de la Suisse: Crédits mixtes, Aide à la balance des paiements, Garantie contre les risques à l'exportation, Berne, 1989, chap. III.2

9. Message du 19-2-1986, pp.143-146; Message du 21-2-1990, pp.132-136.

10. La Statistique du commerce extérieur de la Suisse montre que les exportations de pièces détachées de véhicules (position 8706.34) à destination du Ghana sont tombées de 6,2 millions de francs (niveau moyen 1984-86) à 0,1 million en 1987.

11. Le problème général des fonds de contrevaleur résultant des aides à la balance des paiements est clairement présenté au point 343 du Message concernant la continuation du financement de mesures de politique économique et commerciale, du 21-2-1990. L'analyse concerne aussi la contribution suisse au PAMSCAD.

12. On pourrait toutefois penser qu'un lien discret a encore favorisé les fournisseurs suisses dans le cadre de l'aide IV: l'affectation de celle-ci au paiement de factures libellées en francs suisses. Notons que des firmes étrangères établissent parfois aussi des factures dans notre monnaie. D'autre part, comme le montant de l'aide était bien inférieur aux achats totaux du Ghana à des fournisseurs suisses, cette procédure pouvait difficilement provoquer des distorsions de concurrence.

13. Message concernant la continuation du financement de mesures de politique économique et commerciale, du 21-2-1990, p. 135.

14. OFAEE, Mesures de désendettement entreprises par la Suisse, 6-2-1992, p. 4.

15. Coopération au développement de la Confédération Suisse, Rapport 1991, p. 6. 


\section{Zusammenfassung}

Die Schweiz sieht Strukturanpassungsprogramme derzeit als eine geeignete Strategie zur Überwindung der Wirtschaftskrise in den Entwicklungsländern an. Sie hat vor allem in den afrikanischen Ländern südlich der Sahara an solchen Programmen teilgenommen. Die Studie über die Erfahrung Ghanas soll zur Reflexion betreffend die Wirksamkeit der Strukturanpassungsprogramme beitragen.

Zwischen 1984 und 1992 hat der Bund 87 Millionen Franken für den wirtschaftliche Wiederaufbau Ghanas gezahlt. Die Förderung erfolgte grösstenteils in Form von Zahlungsbilanzhilfen (BAWI) und durch Kofinanzierungen mit der IDA, sowie durch die Finanzierung technischer Hilfe zur Umstrukturierung des Bankensektors (DEH).

Die Schweiz hat sich als eines der ersten Länder bilateral am Strukturanpassungsprogramm für Ghana beteiligt und Ghana war lange Zeit Hauptempfänger der schweizerischen Zahlungsbilanzhilfe. In einer schwierigen Wirtschaftslage hat die Regierung Ghanas selbst Anstrengungen zum Wiederaufbau des Landes unternommen und wurde dabei von der internationalen Gemeinschaft unter Führung von IWF und Weltbank unterstützt. Sie hat es verstanden, Strukturanpassung mit Wirtschaftswachstum zu vereinen.

Konkret hat der Bund Hilfe zur Finanzierung von Einfuhren für die lokale Produktion von Gütern und Dienstleistungen und zur Finanzierung eines Devisenversteigerungssystems geleistet, dessen Gegenwertmittel in lokaler Währung ein Programm zur Armutsbekämpfung finanziert haben. Die von der IDA verwalteten Mittel dienten zur Kofinanzierung der Strukturanpassung des Bankensektors und wurden durch technische Hilfe (Beratung der Zentralbank Ghanas) ergänzt.

Die Finanzierung von Einfuhren zur lokalen Produktion von Gütern und Dienstleistungen hatte insgesamt positive Auswirkungen (ohne grössere Wettbewerbsverzerrungen). Hingegen war die Bereitstellung von Gegenwertmitteln in lokaler Währung für ein Programm zur Armutsbekämpfung nicht zufriedenstellend, weil das Programm schlecht in die Anpassungsstrategie integriert war.

Bezüglich der Reform des Bankensektors lässt sich noch keine Bilanz ziehen. Damit die Banken ihre Rolle spielen, d.h. zur wirtschaftlichen Sanierung beitragen können, müsste die Regierung nach Ansicht des Autors ihre Währungspolitik flexibler gestalten. Betreffend den Finanzsektor Ghanas sollte die Schweiz die Kreditvergabe an Unternehmen fördern, welche wesentliche Güter für den Binnenmarkt und für den Export als Deviseneinnahmequelle produzieren. Hierzu sollte sie die Einrichtung von Entwicklungsfonds fördern und weitere technische Hilfe leisten. 